\title{
TAYLOR THEOREM FOR PLANAR CURVES
}

\author{
ABEDALLAH RABABAH
}

(Communicated by J. Marshall Ash)

\begin{abstract}
We describe an approximation method for planar curves that significantly improves the standard rate obtained by local Taylor approximations. The method exploits the freedom in the choice of the parametrization and achieves the order $4 \mathrm{~m} / 3$ where $m$ is the degree of the approximating polynomial parametrization. Moreover, we show for a particular set of curves that the optimal rate $2 m$ is possible.
\end{abstract}

\section{INTRODUCTION, EXAMPLES, AND MAIN RESULTS}

In computer aided geometric design, curves and surfaces are commonly represented by polynomial parametrizations (cf., e.g., $[1,4,7,15])$. In this paper we describe an approximation procedure for planar curves that significantly improves the standard approximation rate via Taylor's theorem. This method is based on the observation that the parametrization of a curve is not unique and can be suitably modified to improve the approximation order.

Let

$$
\mathscr{C}: t \mapsto\left(\begin{array}{c}
f(t) \\
g(t)
\end{array}\right)
$$

be a regular smooth planar curve (i.e., $\left.\left(f^{\prime}(t), g^{\prime}(t)\right) \neq(0,0)\right)$. We want to approximate $\mathscr{C}$ by a polynomial curve

$$
\mathscr{P}: t \mapsto\left(\begin{array}{c}
X(t) \\
Y(t)
\end{array}\right),
$$

where $X(t)$ and $Y(t)$ are polynomials of degree $m$. If we choose for $X(t)$ and $Y(t)$ the Taylor polynomial of degree $m$, then $\mathscr{P}$ approximates $\mathscr{C}$ with order $m+1$, i.e.,

$$
\{f(t)-X(t)\},\{g(t)-Y(t)\}=\mathscr{O}\left(t^{m+1}\right) .
$$

Surprisingly, a significantly better order is possible. The first result of this type was obtained in [2] by generalization of Hermite interpolation. In addition to position and tangent, the curvature is interpolated at each end point of the

Received by the editors January 21, 1991 and, in revised form, March 5, 1992.

1991 Mathematics Subject Classification. Primary 41A10, 41A25, 41A58.

Key words and phrases. Taylor polynomial, planar curves, approximation order, computer aided geometric design. 
cubic segments, which yields an approximation order of 6 (rather than 4 ). There are more results for special cases in [3, 5, 6, 8-14].

The improvement over the standard order $m+1$ is possible because the parametrization of a curve is not unique. In fact, without loss of generality we may assume that $(f(0), g(0))=(0,0),\left(f^{\prime}(0), g^{\prime}(0)\right)=(1,0)$ so that for small $t$ we can parametrize $\mathscr{C}$ in the form

$$
\mathscr{C}: t \mapsto\left(\begin{array}{c}
X(t) \\
\phi(X(t))
\end{array}\right), \quad t \in \mathbb{R}
$$

for some suitable $\phi(X(t))$.

Remark. The "geometric" distance between the two curves $\mathscr{C}$ and $\mathscr{P}$ is measured in the Hausdorff metric, i.e.,

$$
d(\mathscr{C}, \mathscr{P})=\max \{\max \{\operatorname{dist}(c, \mathscr{P}), c \in \mathscr{C}\}, \max \{\operatorname{dist}(\mathscr{C}, p), p \in \mathscr{P}\}\} .
$$

Since both curves have a common horizontal tangent at the origin, restricted to a sufficiently small neighborhood $U$,

$$
d^{*} \geq d(\mathscr{C} \cap U, \mathscr{P} \cap U) \geq \text { const } \cdot d^{*}
$$

with $d^{*}=\max \{|\phi(X(t))-Y(t)|\}$. The left inequality is trivial, so we must show only the lower bound inequality. Let $\beta$ be the minimal angle so that for any point $p \in(\mathscr{P} \cap U)$ the cone at $p$ contains $\mathscr{P} \cap U$. Let $c_{0}$ and $p_{0}$ be points on $\mathscr{C}$ and $\mathscr{P}$ respectively with the same first coordinate $X(t)$, for which $\left|\overline{c_{0} p_{0}}\right|=d^{*}$. The distance $\left|\overline{c_{0} p_{1}}\right|$ of $c_{0}$ to a closest point $p_{1} \in(\mathscr{P} \cap U)$ is bounded from below by the distance $\left|\overline{c_{0} p_{2}}\right|$ of $c_{0}$ to the cone attached at $p_{0}$. It follows that

$$
d^{*}=\left|\overline{c_{0} p_{0}}\right|=\frac{\left|\overline{c_{0} p_{2}}\right|}{\cos (\beta / 2)} \leq \frac{\left|\overline{c_{0} p_{1}}\right|}{\cos (\beta / 2)} \leq \frac{d}{\cos (\beta / 2)} .
$$

Since the role of $\mathscr{P}$ and $\mathscr{C}$ can be interchanged, the lower bound follows with const $\leq \cos (\beta / 2)$.

Thus, in view of the remark above, $\mathscr{P}$ approximates $\mathscr{C}$ with order $\alpha$, a natural number, iff

$$
\phi(X(t))-Y(t)=\mathscr{O}\left(t^{\alpha}\right)
$$

i.e., iff

$$
\left(\frac{d}{d t}\right)^{j}\{\phi(X(t))-Y(t)\}_{\mid t=0}=0, \quad j=1, \ldots, \alpha-1
$$

and

$$
X(0)=Y(0)=0 \text {. }
$$

If one normalizes the polynomial approximation by choosing $X^{\prime}(0):=1$, then the polynomial approximation is determined by $2 m$ free parameters. Comparing this with the number of equations in (1), leads to the following

Conjecture. A smooth regular planar curve can, in general, be approximated by a polynomial parametrization of degree $\leq m$ with order $2 m$.

We illustrate the conjecture in a special case by constructing a cubic parametrization which approximates with order 6 . To achieve the optimal approximation rate, we have, according to (1), to solve the following nonlinear system of 
equations:

$$
\begin{array}{r}
\phi_{1} X_{1}-Y_{1}=0, \\
\phi_{2} X_{1}^{2}+\phi_{1} X_{2}-Y_{2}=0, \\
\phi_{3} X_{1}^{3}+3 \phi_{2} X_{1} X_{2}+\phi_{1} X_{3}-Y_{3}=0, \\
\phi_{4} X_{1}^{4}+6 \phi_{3} X_{1}^{2} X_{2}+3 \phi_{2} X_{2}^{2}+4 \phi_{2} X_{1} X_{3}=0 \\
\phi_{5} X_{1}^{5}+10 \phi_{4} X_{1}^{3} X_{2}+15 \phi_{3} X_{1} X_{2}^{2}+10 \phi_{3} X_{1}^{2} X_{3}+10 \phi_{2} X_{2} X_{3}=0
\end{array}
$$

where $\phi_{i}=\phi_{i}(X(0)), X_{i}=X_{i}(0)$, and $Y_{i}=Y_{i}(0)$ are the $i$ th derivatives of $\phi$, $X$, and $Y$ respectively. With the normalization $X_{1}=1$ the above nonlinear system reduces to

$$
\begin{aligned}
\phi_{1}-Y_{1} & =0, \\
\phi_{2}+\phi_{1} X_{2}-Y_{2} & =0, \\
\phi_{3}+3 \phi_{2} X_{2}+\phi_{1} X_{3}-Y_{3} & =0, \\
\phi_{4}+6 \phi_{3} X_{2}+3 \phi_{2} X_{2}^{2}+4 \phi_{2} X_{3} & =0, \\
\phi_{5}+10 \phi_{4} X_{2}+15 \phi_{3} X_{2}^{2}+10 \phi_{3} X_{3}+10 \phi_{2} X_{2} X_{3} & =0 .
\end{aligned}
$$

The general structure of the nonlinear system now becomes clear. The last two equations involve only the parameters $X_{2}$ and $X_{3}$. Once those are determined, one can substitute their values into the first three equations, which yields the remaining parameters $Y_{1}, Y_{2}$, and $Y_{3}$.

Assuming that $\phi_{2} \neq 0$, we can solve for $X_{3}$ in the fourth equation,

$$
X_{3}=\frac{-1}{4 \phi_{2}}\left(\phi_{4}+6 \phi_{3} X_{2}+3 \phi_{2} X_{2}^{2}\right) \text {. }
$$

Substituting in the last equation yields

$$
\begin{gathered}
\phi_{5}+10 \phi_{4} X_{2}+15 \phi_{3} X_{2}^{2}-\frac{10 \phi_{3}}{4 \phi_{2}}\left(\phi_{4}+6 \phi_{3} X_{2}+3 \phi_{2} X_{2}^{2}\right) \\
-\frac{10 \phi_{2} X_{2}}{4 \phi_{2}}\left(\phi_{4}+6 \phi_{3} X_{2}+3 \phi_{2} X_{2}^{2}\right)=0,
\end{gathered}
$$

and after simplifications

$$
\begin{gathered}
\left(\phi_{5}-\frac{5 \phi_{3} \phi_{4}}{2 \phi_{2}}\right)+\left(10 \phi_{4}-\left(\frac{30 \phi_{3}^{2}+5 \phi_{2} \phi_{4}}{2 \phi_{2}}\right)\right) X_{2} \\
+\left(15 \phi_{3}-\frac{45 \phi_{2} \phi_{3}}{2 \phi_{2}}\right) X_{2}^{2}-\left(\frac{15 \phi_{2}}{2}\right) X_{2}^{3}=0 .
\end{gathered}
$$

Since the highest power of $X_{2}$ is odd (assuming $\phi_{2} \neq 0$ ), this equation always has at least one solution. Hence, under the assumption $\phi_{2} \neq 0$ the nonlinear system can be solved, which confirms the conjecture in this case.

If, on the other hand, $\phi_{2}=0$, then a solution need not exist. Consider the following cases:

(a) $\phi_{3}=0$. From the fourth equation we get $\phi_{4}=0$ and so there is no solution.

(b) $\phi_{4}=0$. From the fourth equation, $\phi_{3}=0$ or $\phi_{3}=X_{2}=0$ or $X_{2}=0$; for the first two cases, there is no solution, for the third case, we get only a solution for $X_{3}=-\phi_{5} / 10 \phi_{3}$ where $\phi_{3} \neq 0$. 
(c) $\phi_{3}, \phi_{4}, \phi_{5} \neq 0$. We have a solution for $X_{2}$ and $X_{3}$. So, the condition $\phi_{2} \neq 0$ is sufficient but not necessary to get a solution. This shows that in general some generic conditions on the Taylor coefficients of $\phi$ are necessary.

While we cannot prove the above conjecture in general, we can solve the first $4 \mathrm{~m} / 3$ equations by an appropriate choice of the derivatives of $X$. This yields the following result, which still provides a considerable improvement over the rate of the Taylor approximation.

Theorem 1. For $m>3$, define

$$
n_{1}= \begin{cases}n & \text { for } m=3 n \text { or } 3 n+1, \\ n+1 & \text { for } m=3 n+2 .\end{cases}
$$

Then for almost all $\left(\phi_{1}, \phi_{2}, \ldots, \phi_{m+n_{1}}\right) \in \mathbb{R}^{m+n_{1}}$ there is a solution for the first $m+n_{1}$ equations in (1).

Moreover, we confirm the conjecture for a special case by finding a set of Taylor coefficients $\left(\phi_{1}, \ldots, \phi_{2 m-1}\right)$ of nonzero measure for which the optimal approximation of order $2 m$ is attained. To this end, we view equations $m+1$, $m+2, \ldots, 2 m-1$ in (1) as a nonlinear system

$$
F(\Phi, V)=\left(\frac{d}{d t}\right)^{l} \phi(X(t))_{\mid t=0}=0, \quad l=m+1, m+2, \ldots, 2 m-1,
$$

with $V:=\left(X_{2}, \ldots, X_{m}\right), X_{1}=1$, and $\Phi:=\left(\phi_{2}, \ldots, \phi_{2 m-1}\right)$ and show that this system is solvable in a neighborhood of a particular solution $\left(\Phi^{*}, X^{*}\right)$.

Theorem 2. Define $X_{j}^{*}=0, j=2, \ldots, m$, and $\phi_{j}^{*}=1$ for $j=m$, and $=0$ otherwise. Then $\left(\Phi^{*}, X^{*}\right)$, where $X^{*}=\left(X_{2}^{*}, \ldots, X_{m}^{*}\right)$ and $\Phi^{*}=\left(\phi_{2}^{*}, \ldots\right.$, $\left.\phi_{2 m-1}^{*}\right)$, is a solution of $F(\Phi, V)=0$. Moreover, there exists a neighborhood of $\Phi^{*}$ such that the nonlinear system (1) is uniquely solvable.

\section{Proofs}

For the proofs of Theorems 1 and 2, the following formula for the derivatives of $\phi(X(t))$ will be needed.

For $l=1, \ldots, 2 m-1$

$$
\left(\frac{d}{d t}\right)^{l}\{\phi(X(t))\}_{\mid t=0}=\sum_{\substack{i=1 \\ p_{1}+\cdots+p_{i}=l}}^{l} c\left(i, l \mid p_{1}, \ldots, p_{i}\right) \phi_{i} X_{p_{1}} \cdots X_{p_{i}}
$$

where $\phi_{i}$ and $X_{i}$ are the $i$ th derivatives of $\phi$ and $X$ respectively, and $c\left(i, l \mid p_{1}, \ldots, p_{i}\right)=c(i, l)$ is a strictly positive constant which depends on $i, p_{1}, \ldots, p_{i}$, and $l$. Notice that in (2) for $l>m$ the first term in the sum, $i=1$, is cancelled because it involves the derivative $X_{l}$, and this is zero when $l>m$.

2.1. Proof of Theorem 1. In order to prove Theorem 1, we have to solve (1) for $j=1, \ldots, m+n_{1}$. As we already pointed out earlier, this means that we have to solve equations $m+1, m+2, \ldots, m+n_{1}$ for $X_{j}, j=m$, $m-1, \ldots, m-n_{1}+1$, and then determine $Y_{i}, i=1, \ldots, m$, from equations $1,2, \ldots, m$. 
We choose

$$
X_{1}=1, X_{2}=\cdots=X_{m-n_{1}}=0
$$

and assume that $\phi_{2}, \ldots, \phi_{2 n_{1}} \neq 0$. Then only terms of the form

$$
c(p, s) \phi_{l-p+1} X_{1}^{l-p} X_{p}=c(p, s) \phi_{l-p+1} X_{p} \quad \text { where } m-n_{1}+1 \leq p \leq m
$$

or

$$
c(s) \phi_{l} X_{1}^{l}=-b_{m-s+1}
$$

are nonzero in the $(m+s)$ th equation in $(2)$, where $1 \leq s \leq n_{1}$. Indeed, for a term with more than two different factors in $X$, one of which is $X_{1}$, i.e., a term of the form

$$
c(p, s) \phi_{l-p_{1}-p_{2}+2} X_{1}^{l-p_{1}-p_{2}} X_{p_{1}} X_{p_{2}},
$$

we have $p_{1}+p_{2} \leq l \leq m+n_{1}$; yet if this term should be nonzero then $p_{1}$, $p_{2} \geq m-n_{1}+1$ and thus $p_{1}+p_{2} \geq 2 m-2 n_{1}+2$. Hence $2 m-2 n_{1}+2 \leq m+n_{1}$ and so $m+2 \leq 3 n_{1}$, which contradicts the definition of $n_{1}$. Hence, substituting (3) in (2) we get the following linear system of equations

$$
\left(\frac{d}{d t}\right)^{m+s}\{\phi(X(t))\}_{\mid t=0}=-b_{m-s+1}+\sum_{r=1}^{n_{1}} c(r, s) \phi_{r+s} X_{m-r+1}, \quad s=1, \ldots, n_{1} \text {. }
$$

This may be rewritten in matrix form $A D=b$, where

$$
\begin{gathered}
A=\left[\begin{array}{cccc}
c(1,1) \phi_{2} & c(2,1) \phi_{3} & \cdots & c\left(n_{1}, 1\right) \phi_{n_{1}+1} \\
c(1,2) \phi_{3} & c(2,2) \phi_{4} & \cdots & c\left(n_{1}, 2\right) \phi_{n_{1}+2} \\
\vdots & \vdots & \ddots & \vdots \\
c\left(1, n_{1}\right) \phi_{n_{1}+1} & c\left(2, n_{1}\right) \phi_{n_{1}+2} & \cdots & c\left(n_{1}, n_{1}\right) \phi_{2 n_{1}}
\end{array}\right] \\
D=\left[\begin{array}{c}
X_{m} \\
X_{m-1} \\
\vdots \\
X_{m-n_{1}+1}
\end{array}\right], \quad b=\left[\begin{array}{c}
b_{m} \\
b_{m-1} \\
\vdots \\
b_{m-n_{1}+1}
\end{array}\right]
\end{gathered}
$$

To show that $A D=b$ can be solved, it is sufficient to show that $\operatorname{det}(A) \neq 0$.

To this end, we notice that for an invertible matrix $B$ the two partitioned matrices

$$
\left[\begin{array}{ll}
B & c \\
d & e
\end{array}\right] \text { and }\left[\begin{array}{cc}
B & c \\
0 & e-d B^{-1} c
\end{array}\right]
$$

are equivalent and hence, for fixed $B$, fixed row matrix $d$, and fixed column matrix $c$, are both invertible except for one choice for the number $e$. In the present application, the element of the $j$ th upper left principal submatrix of $A$ corresponding to $e$ is of the form $c(j, j) \phi_{2 j}$ and is the only element of that submatrix involving $\phi_{2 j}$. Hence, the matrix $A$ is invertible and the system $A D=b$ has a solution. The proof of Theorem 1 is complete.

2.2 Proof of Theorem 2. For the proof of Theorem 2, we will apply the implicit function theorem. We will verify that $\left(\Phi^{*}, X^{*}\right)$ is a particular solution and show that the Jacobi matrix $F_{V}\left(\Phi^{*}, X^{*}\right)$ is invertible. Hence, we can write $X$ as a function of $\Phi$ near $\Phi=\Phi^{*}$.

Recall that

(4) $\phi_{m}=1, \quad X_{1}=1, \quad \phi_{\mu}=0, \quad \mu=2, \ldots, m-1, m+1, \ldots, 2 m-1$. 
Substituting (4) in (2) for $l=m+1, m+2, \ldots, 2 m-1$, the sum involves only terms of the form

$$
c \phi_{m} X_{p_{1}} \cdots X_{p_{m}}, \quad p_{1}+p_{2}+\cdots+p_{m}=l .
$$

More precisely, we have for

$$
l=m+1 \text { : }
$$

(i) $p_{1}=p_{2}=\cdots=p_{m-1}=1$ and $p_{m}=2$;

$l=m+2$ :

(i) $p_{1}=p_{2}=\cdots=p_{m-1}=1$ and $p_{m}=3$,

(ii) $p_{1}=p_{2}=\cdots=p_{m-2}=1$ and $p_{m-1}=p_{m}=2$;

in general for $s=3,4, \ldots, m-1$ we have for

$l=m+s$ :

(i) $p_{1}=p_{2}=\cdots=p_{m-1}=1$ and $p_{m}=s+1$,

(ii) $p_{1}=p_{2}=\cdots=p_{m-2}=1, p_{m-1}=2$, and $p_{m}=s$,

(iii) $p_{1}=p_{2}=\cdots=p_{m-3}=1, p_{m-2}=p_{m-1}=2$, and $p_{m}=s-1$,

(iv) $p_{1}=p_{2}=\cdots=p_{m-4}=1, p_{m-3}=p_{m-2}=p_{m-1}=2$, and $p_{m}=s-2$,

and so on.

Therefore, substituting (4) into (2), we get the system

$$
F\left(\Phi^{*}, V\right)=A V=0,
$$

where

$$
\begin{gathered}
A=\left[\begin{array}{ccccc}
0 & 0 & \cdots & 0 & c(m-1,1) X_{1}^{m-1} \\
0 & 0 & \cdots & c(m-2,2) X_{1}^{m-1} & A_{m-1,2} \\
0 & 0 & \ddots & A_{m-2,3} & A_{m-1,3} \\
\vdots & \vdots & & \vdots & \vdots \\
0 & c(2, m-2) X_{1}^{m-1} & \cdots & A_{m-2, m-2} & A_{m-1, m-2} \\
c(1, m-1) X_{1}^{m-1} & A_{2, m-1} & \cdots & A_{m-2, m-1} & A_{m-1, m-1}
\end{array}\right], \\
V=\left[\begin{array}{c}
X_{m} \\
X_{m-1} \\
\vdots \\
X_{2}
\end{array}\right],
\end{gathered}
$$

and

$$
A_{r, s}= \begin{cases}0 & \text { for } r+s<m, \\ c(r, s) X_{1}^{m-1} & \text { for } r+s=m, \\ c(r, s) X_{1}^{2 m-(r+s+1)} X_{2}^{r+s-m} & \text { for } m<r+s<2 m-2 .\end{cases}
$$

This system has the solution $V=X^{*}$ with

$$
X_{2}^{*}=X_{3}^{*}=\cdots=X_{m}^{*}=0 \text {. }
$$

We now compute the Jacobian at the points $\Phi^{*}=\left(\phi_{2}^{*}, \phi_{3}^{*}, \ldots, \phi_{2 m-1}^{*}\right)$ and $X^{*}=\left(X_{2}^{*}, X_{3}^{*}, \ldots, X_{m}^{*}\right)$ with $\phi_{m}^{*}=1, \phi_{\mu}^{*}=0, \mu=2, \ldots, m-1, m+$ $1, \ldots, 2 m-1$, and $X_{j}^{*}=0, j=2, \ldots, m$.

Since

$$
F\left(\Phi^{*}, V\right)=A\left(\Phi^{*}, V\right) \cdot V
$$


we have

$$
F_{V}\left(\Phi^{*}, V\right)=A_{V}\left(\Phi^{*}, V\right) \cdot V+A\left(\Phi^{*}, V\right)
$$

So

$$
F_{V}\left(\Phi^{*}, X^{*}\right)=A\left(\Phi^{*}, X^{*}\right) .
$$

The Jacobi matrix at $\left(\Phi^{*}, X^{*}\right)$ is

$$
\left[\begin{array}{ccccc}
0 & 0 & \cdots & 0 & c(m-1,1) \\
0 & 0 & \cdots & c(m-2,2) & 0 \\
0 & 0 & . & 0 & 0 \\
\vdots & \vdots & & \vdots & \vdots \\
0 & c(2, m-2) & \cdots & 0 & 0 \\
c(1, m-1) & 0 & \cdots & 0 & 0
\end{array}\right]
$$

and so

$$
\begin{aligned}
\operatorname{det}\left(F_{V}\left(\Phi^{*}, X^{*}\right)\right)= & c(m-1,1) \times c(m-2,2) \\
& \times \cdots \times c(2, m-2) \times c(1, m-1),
\end{aligned}
$$

which shows that the Jacobi matrix is trivially invertible. Hence, the hypotheses of the implicit function theorem are satisfied at the point $\left(\Phi^{*}, X^{*}\right)$, which completes the proof of Theorem 2 .

\section{ACKNOWLEDGMENT}

I gratefully acknowledge the support of Professor Klaus Höllig of the Department of Mathematics at Universität Stuttgart, who brought the conjecture discussed in this paper to my attention. The final version of the paper reflects several helpful comments of the referee for which I am very grateful.

\section{REFERENCES}

1. W. Beohm, G. Farin, and J. Kahmann, A survey of curve and surface methods in CAGD, Computer Aided Geometric Design 1 (1984), 1-60.

2. C. de Boor, K. Höllig, and M. Sabin, High accuracy geometric Hermite interpolation, Computer Aided Geometric Design 4 (1988), 269-278.

3. T. Dokken, M. Dxhlen, T. Lyche, and K. Mørken, Good approximation of circles by curvature-continuous Bézier curves, Computer Aided Geometric Design 7 (1990), 33-41.

4. G. Farin, Curves and surfaces for computer aided geometric design, Academic Press, Boston, MA, 1988.

5. M. Goldapp, Approximation of circular arces by cubic polynomials, Computer Aided Geometric Design 8 (1991), 227-238.

6. T. N. T. Goodman and K. Unsworth, Shape preserving interpolation by curvature continuous parametric curves, Computer Aided Geometric Design 5 (1988), 323-340.

7. J. Hoschek and D. Lasser, Grundlagen der geometrischen Datenverarbeitung, Teubner, Stuttgart, 1989.

8. K. Höllig, Algorithms for rational spline curves, Conference on Applied Mathematics and Computing, ARO Report 88-1, 1988.

9. S. Pumm, Interpolation an "zu vielen" Punkten, Diplomarbeit, Mathematisches Institut A, Stuttgart Universität, Pfaffenwaldring 57, 7000 Stuttgart 80, Germany, 1990.

10. A. Rababah, Approximation von Kurven mit Polynomen und Splines, Ph.D. Thesis, Mathematisches Institut A, Universität Stuttgart, Pfaffenwaldring 57, D-7000 Stuttgart 80, Germany, 1992. 
11. _ High accuracy piecewise approximation for planar curves, J. Approx. Theory (to appear).

12. __ High order approximation method for curves, Comput. Aided Geom. Design (to appear).

13. _ Hermite approximation with high accuracy, Constr. Approx. (to appear).

14. T. W. Sederberg and M. Kakimoto, Approximating rational curves using polynomial curves, Technical Report ECGL 90-03, 1990.

15. F. Yamaguchi, Curves and surfaces in computer aided geometric design, Springer, New York, 1988.

Mathematisches Institut A, Universität Stuttgart, Pfaffenwaldring 57, 7000 StutTGart 80, Germany

E-mail address: rababah@mathematik.uni-stuttgart.de 